\title{
Cognitive and weight-related correlates of flexible and rigid restrained eating behaviour
}

\author{
Joachim Westenhoefer ${ }^{1}$, Daniel Engel ${ }^{1}$, Claus Holst ${ }^{2}$, Jürgen Lorenz ${ }^{3}$, Matthew Peacock ${ }^{5}$, James \\ Stubbs $^{4}$, Stephen Whybrow ${ }^{5}$, Monique Raats ${ }^{5}$
}

${ }^{1}$ Hamburg University of Applied Sciences, Department of Health Sciences, Germany

${ }^{2}$ Institute of Preventive Medicine, Copenhagen, Denmark

${ }^{3}$ Hamburg University of Applied Sciences, Department of Biomedical Engineering, Germany

${ }^{4}$ Slimming World, Alfreton, UK

${ }^{5}$ University of Surrey, Guildford, UK

Corresponding author:

Prof. Dr. Joachim Westenhoefer; Hamburg University of Applied Sciences; Department Health

Sciences; Lohbruegger Kirchstrasse 65; 21033 Hamburg, Germany; Email: joachim@westenhoefer.de 


\begin{abstract}
Objectives: Examine the association between components of restrained eating, cognitive performance and weight loss maintenance.

Methods: 106 women, all members of a commercial slimming organisation for at least 6 months (mean \pm SD: $15.7 \pm 12.4$ months), were studied who, having lost $10.1 \pm 9.7 \mathrm{~kg}$ of their initial weight, were hoping to sustain their weight loss during the 6 month study. Dietary restraint subcomponents flexible and rigid restraint, as well as preoccupying cognitions with food, body-shape and diet were assessed using questionnaires. Attentional bias to food and shape-related stimuli was measured using a modified Stroop test. Working memory performance was assessed using the N-back test. These factors, and participant weight, were measured twice at 6 month intervals.

Results: Rigid restraint was associated with attentional bias to food and shape-related stimuli $(r=$ $0.43, p<0.001$ resp. $r=0.49, p<0.001)$ whereas flexible restraint correlated with impaired working memory $(r=-0.25, p<0.05)$. In a multiple regression analyses, flexible restraint was associated with more weight lost and better weight loss maintenance, while rigid restraint was associated with less weight loss.

Conclusions: Rigid restraint correlates with a range of preoccupying cognitions and attentional bias to food and shape-related stimuli. Flexible restraint, despite the impaired working memory performance, predicts better long-term weight loss. Explicitly encouraging flexible restraint may be important in preventing and treating obesity.
\end{abstract}

Keywords: restrained eating, cognitive performance, weight loss 


\section{Introduction}

Obesity has become one of the western worlds' most serious public health problems. A key contributor to rising obesity is the passive overconsumption of high-fat and energy-dense food (Viskaal-van Dongen, de Graaf, Siebelink, \& Kok, 2009). Attempts to counter this by eating less food are known as "restrained eating" or "dietary restraint" (Herman \& Polivy, 1975). These are typically only partially successful because most weight lost is later regained (Saris \& Harper, 2005).

Crucially, dietary restraint depends on psychological processes such as self-awareness and selfmonitoring which impose cognitive demands on the dieter. Indeed, various studies have correlated dieting with impairments of mental performance, in particular slower responding in attention tasks and poorer working memory performance (Green \& Rogers, 1998; Vreugdenburg, Bryan, \& Kemps, 2003).

These impairments have potential consequences for dieting: (i) Functional impairments could be experienced as an additional cost to dieting which makes the dieter more likely to give up. (ii) Deficits in attention and memory might impair the dieter's crucial (Burke, Wang, \& Sevick, 2011) ability to monitor and control food intake.

To our knowledge, the associations of such cognitive effects have only been examined with regard to eating disorders, dieting or restrained eating. Restrained eating, however, emerges as a heterogeneous construct (Westenhoefer, 1991; Westenhoefer, Stunkard, \& Pudel, 1999) encompassing two important subcomponents, namely rigid and flexible restraint.

Rigid restraint is characterized by a dichotomous, all-or-nothing approach and a tendency to oscillate between periods of strict dieting and periods in which the "fattening" foods are freely consumed. Flexible restraint is a more graduated approach to losing weight in which "fattening" foods are permitted in limited quantities rather than avoided entirely, leading to more consistent and sustainable dieting.

These two components predict very different outcomes. Whilst rigid restraint is associated with a greater tendency to overeat and poorer weight control, flexible restraint predicts better weight control and reduced overeating (Westenhoefer et al., 1999; Westenhoefer, von Falck, Stellfeldt, \& Fintelmann, 2004). It is currently unknown whether either or both components cause the cognitive impairments associated with dietary restraint.

The present study will therefore investigate (i) the relationship between flexible/rigid restraint patterns and cognitive performance, and (ii) the relationship between these components and sustainable weight loss.

\section{Methods}

106 volunteers aged 21-74 were recruited, all active members of a commercial UK slimming organization (www.slimmingworld.com) for at least 6 months. Only women were recruited because membership is overwhelmingly female.

To measure attentional bias we used a modified Stroop test (Stroop, 1935). Variants of this task are widely used to study perceptual-cognitive interference by comparing participants' response speed to the colour of emotionally salient words with that to neutral words (Field \& Cox, 2008). The delay in response to salient stimuli is called "attentional bias" (Dobson \& Dozois, 2004; Field \& Cox, 2008).

Participants with eating disorders consistently exhibit attentional bias when processing diet and body-shape-related words (Dobson \& Dozois, 2004) so it seems reasonable to suggest that the same may be true of obese and dieting participants (Green \& Rogers, 1993), for whom these words are also resonant.

Our modified Stroop task asked participants whether a succession of words on a monitor were adjectives or nouns, forcing them to read the word instead of responding only to its colour. 48 
neutral words and $48 \mathrm{diet} /$ body-shape-related words were presented randomly on a computer screen. Neutral and diet/shape-related words did not differ significantly in number of characters, syllables and the word frequency ranks, based on Davies (2012). We measured attentional bias via the differences in reaction time between diet/body-shape-related words and neutral words.

We used the N-back task to measure working memory speed and accuracy (Owen, McMillan, Laird, \& Bullmore, 2005). This presents a series of letters on a screen and asks the participant to indicate, via yes/no response pad buttons, whether the current letter matches one shown $\mathrm{N}$ trials ago. For example, in the "2-back" version the current letter is compared that shown 2 trials ago. We used both "1-back" (low demand) and "2-back" (high demand) conditions.

Participants completed a 20 item questionnaire which assesses preoccupation with weight loss (Vreugdenburg et al., 2003). From this a total score for preoccupying cognitions (Total PC) and subscale scores for food, shape and diet-related PC were calculated.

In addition, the participants completed the Eating Inventory (Stunkard \& Messick, 1985), and additional items assessing both flexible (12 items) and rigid restraint (16 items) of eating behaviour.

The Cognitive Performance Tests and Questionnaires were administered to each participant first on Investigation day 1 (ID1) and then 6 months later on Investigation day 2 (ID2). Trials took place at participants' homes because it was impractical for all volunteers to travel to the laboratory. On both occasions participants were tested at the same time of day, alone and in quiet conditions to minimise distracters.

Weight (in $\mathrm{kg}$ ) and height (in $\mathrm{m}$ ) were measured at ID1 and ID2 and Body Mass Index (BMI) calculated $\left(\mathrm{BMI}=\mathrm{kg} / \mathrm{m}^{2}\right)$. Relative weight change was calculated as weight change divided by ID1 weight ((kg (ID2) - kg (ID1)) / kg (ID1)). Weight history records were obtained from Slimming World whenever possible, enabling us to identify starting date and initial weight of 68 and 74 participants respectively, and participant weight 6 months prior to ID1 for 84 participants.

Statistical analyses were carried out using SPSS (Versions 18 and 20). Descriptive statistics are given as mean \pm standard deviation. The significance level was set to $\alpha=0.05$. Differences between pairs of means were tested using Student's t-test. Correlations between variables were investigated using Person's correlation coefficient. In addition, multiple regression analyses (MRAs) were computed to examine the relationship between a dependent variable and several independent variables.

The study protocol was approved by the Grampian Health Ethics Committee.

\section{Results}

106 participants took weight and cognitive performance tests on ID1. Complete follow-up data (body weight, cognitive tests and questionnaire data) for 76 participants were collected at ID2. On ID1 participants had an average age of $45.7 \pm 11.8$ years and a BMI of $30.3 \pm 5.4$. Average scores of flexible and rigid restraint were $5.3 \pm 2.7$ and $7.7 \pm 2.8$. Between ID1 and ID2 weight change ranged from a weight loss of $12.4 \mathrm{~kg}$ to a weight gain of $7.6 \mathrm{~kg}$. However, average weight and BMI for ID1 and ID2 were not significantly different $(p>0.05)$, nor was relative weight change between ID1 and ID2 statistically significant $(p>0.05)$.

Average membership duration prior to ID1 was $15.7 \pm 12.4$ months (median $=12$.). During that time participants had lost an average of $10.1 \pm 9.7 \mathrm{~kg}$. In the six months prior to ID1 participants lost an average of $2.8 \pm 6.5 \mathrm{~kg}(n=84)$.

Flexible and rigid restraint were moderately correlated $(r=0.47 ; p<0.001$ on ID1; $r=0.40 ; p<$ 0.001 on ID2). Their average scores did not differ significantly between ID1 and ID2 ( $p>0.2)$. 
BMI on ID1 was significantly predicted using MRA $\left(R^{2}=0.11 ; p<0.01\right)$ with flexible restraint significantly associated with lower $\mathrm{BMI}(\beta=-0.31 ; p<0.005)$, rigid restraint with higher $\mathrm{BMI}(\beta=0.22$; $p<0.05)$. Age was not a significant predictor $(\beta=0.14 ; p>0.1)$.

Similarly, weight change since joining Slimming World was significantly predicted (adjusted $R^{2}=$ 0.36; $p<0.001$ ): Flexible restraint and weight on joining were associated with greater weight loss ( $\beta=$ $0.32 ; p<0.005$ resp. $\beta=0.58 ; p<0.001)$, rigid restraint with less weight loss $(\beta=-0.30 ; p<0.005)$. Age was again not significant $(\beta=0.02 ; p=0.85)$.

A stepwise MRA using relative weight change over the study period as dependent variable and flexible restraint, rigid restraint, age and BMI on ID1 as predictors produced a solution $\left(R^{2}=0.06 ; p<\right.$ $0.05)$ including only flexible restraint as a significant predictor $(\beta=-0.24 ; p<0.05)$ of greater weight loss.

Flexible and rigid restraint of eating behaviour were related to preoccupying cognitions regarding shape, food and diet (see table 1). Rigid restraint was positively correlated to total score for preoccupying cognitions and all three sub-components, whereas flexible restraint was associated with diet-related preoccupying cognitions, but not with shape or food-related cognitions.

In the ID1 attentional bias test average reaction time to neutral stimuli was $968 \pm 155 \mathrm{~ms}$ and average reaction time to (positive) food/weight-related stimuli was $997 \pm 167 \mathrm{~ms}(\mathrm{t}=-4.3 ; \mathrm{p}<0.001$ ). The difference between positive and neutral stimuli was significantly correlated to rigid restraint $(r=$ $0.27 ; p<0.01)$, but not to flexible restraint $(r=0.10 ; p>0.3)$. On ID2, average reaction times became significantly faster for both neutral $(918 \pm 139 \mathrm{~ms} ; \mathrm{t}=2.29 ; \mathrm{p}<0.05)$ and positive stimuli $(943 \pm 158$ $\mathrm{ms} ; \mathrm{t}=2.93 ; \mathrm{p}<0.01$ ) but reaction time differences did not correlate significantly with restraint subtypes ( $r=-0.08, p>0.5$ for rigid restraint; $r=0.23, p=0.052$ for flexible restraint). The number of correct responses did not differ significantly between neutral and positive stimuli or different subtypes of restraint.

On the ID1 N-back test flexible restraint correlated significantly with fewer correct responses in the 2-back task (see table 2).On ID2 both response times and number of correct responses improved significantly for the 2-back task $(t=3.05 ; p<0.01, t=-2.96 ; p<0.01)$. There were no significant correlations between dietary restraint subtypes and working memory performance.

\section{Discussion}

This study investigated cognitive and weight-related correlates of flexibly and rigidly restrained eating in long-term (mean 15.7 months) members of a commercial slimming club.

Firstly, the results support other studies (Westenhoefer u. a., 1999, 2004) linking higher flexible restraint with lower BMI and greater weight loss, both from the beginning of slimming club membership and over the study period. Rigid restraint correlated with higher BMI and poorer weight loss.

Secondly, this study showed that flexible and rigid restraint correlate with different sets of preoccupying cognitions. Flexible restraint correlated only with diet-related preoccupations which reflect the awareness of the sugar, fat and energy content, which may actually help weight management. Rigid restraint's association with the whole range of diet, food and shape-related preoccupations may be related to the development of food cravings and their role in diet failure (Hill, 2007; Meule, Westenhöfer, \& Kübler, 2011a).

Thirdly, this study found that rigid restraint predicts slower responses to food/shape-related stimuli than neutral stimuli. This suggests that rigid restraint correlates also with an increased cognitive burden for food-related information. This could make sustained dieting difficult.

Finally, this data suggests that flexible dietary restraint is associated with poorer working memory. Reported differences in memory performance between dieters and non-dieters (Green \& 
Rogers, 1998; Vreugdenburg et al., 2003) may therefore stem from the flexible restraint component of dietary restraint. We cannot currently determine whether this is due to the resultant negative energy balance or the cognitive burden of having to continuously monitor and evaluate food intake.

Limitations of these results include an increased risk of error arising from conducting the cognitive tests in homes rather than a laboratory, though this is unlikely to have resulted in systematic bias and may actually increase external and ecological validity. Also, comparisons of ID1 and ID2 cognitive test results suggest learning effects which may obscure relevant response patterns shifts.

In summary, this study showed that flexible dietary restraint predicts better and more sustainable weight loss. Whilst rigid restraint is associated with a range of cognitive preoccupations and slower processing of food and shape-related stimuli, flexible restraint correlates with working memory impairments. Future research must investigate the processes underlying these associations and explore their implications. We recommend that weight loss interventions discourage rigid restraint of eating behaviour and instead foster the development of flexible dietary restraint, whilst researchers investigate how to reduce the cognitive demands of continuous and effortful self-control on dieters. 


\section{References}

Davies, M. (2012, January 24). Word frequency data from the Corpus of Contemporary American English. Retrieved from http://www.wordfrequency.info

Dobson, K. S., \& Dozois, D. J. (2004). Attentional biases in eating disorders: a meta-analytic review of Stroop performance. Clin Psychol Rev, 23(8), 1001-22. doi:S027273580300093X [pii]

Field, M., \& Cox, W. M. (2008). Attentional bias in addictive behaviors: a review of its development, causes, and consequences. Drug and Alcohol Dependence, 97(1-2), 1-20. doi:10.1016/j.drugalcdep.2008.03.030

Green, M. W., \& Rogers, P. J. (1993). Selective attention to food and body shape words in dieters and restrained nondieters. Int J Eat Disord, 14(4), 515-7.

Green, M. W., \& Rogers, P. J. (1998). Impairments in working memory associated with spontaneous dieting behaviour. Psychol Med, 28(5), 1063-70.

Herman, C. P., \& Polivy, J. (1975). Anxiety, restraint, and eating behavior. J Abnorm Psychol, 84(6), 66-72.

Hill, A. J. (2007). The psychology of food craving. The Proceedings of the Nutrition Society, 66(2), 277-285. doi:10.1017/S0029665107005502

Meule, A., Westenhöfer, J., \& Kübler, A. (2011). Food cravings mediate the relationship between rigid, but not flexible control of eating behavior and dieting success. Appetite, 57(3), 582-584. doi:10.1016/j.appet.2011.07.013

Owen, A. M., McMillan, K. M., Laird, A. R., \& Bullmore, E. (2005). N-back working memory paradigm: a meta-analysis of normative functional neuroimaging studies. Hum Brain Mapp, 25(1), 46-59. doi:10.1002/hbm.20131

Saris, W. H., \& Harper, A. (2005). DiOGenes: a multidisciplinary offensive focused on the obesity epidemic. Obes Rev, 6(2), 175-6. doi:OBR200 [pii] 10.1111/j.1467-789x.2005.00200.x

Stroop, J. R. (1935). Studies of interference in serial verbal reactions. Journal of Experimental Psychology.

Stunkard, A. J., \& Messick, S. (1985). The three-factor eating questionnaire to measure dietary restraint, disinhibition and hunger. J Psychosom Res, 29(1), 71-83.

Viskaal-van Dongen, M., de Graaf, C., Siebelink, E., \& Kok, F. J. (2009). Hidden fat facilitates passive overconsumption. J Nutr, 139(2), 394-9. doi:jn.108.096123 [pii] 10.3945/jn.108.096123 [doi]

Vreugdenburg, L., Bryan, J., \& Kemps, E. (2003). The effect of self-initiated weight-loss dieting on working memory: the role of preoccupying cognitions. Appetite, 41(3), 291-300.

doi:S0195666303001077 [pii]

Westenhoefer, J. (1991). Dietary restraint and disinhibition: is restraint a homogeneous construct? Appetite, 16(1), 45-55.

Westenhoefer, J., Stunkard, A. J., \& Pudel, V. (1999). Validation of the flexible and rigid control dimensions of dietary restraint. Int J Eat Disord, 26(1), 53-64. doi:10.1002/(SICI)1098108X(199907)26:1<53::AID-EAT7>3.0.CO;2-N [pii]

Westenhoefer, J., von Falck, B., Stellfeldt, A., \& Fintelmann, S. (2004). Behavioural correlates of successful weight reduction over 3 y. Results from the Lean Habits Study. Int J Obes Relat Metab Disord, 28(2), 334-5. doi:10.1038/sj.ijo.0802530 0802530 [pii] 


\section{Tables}

Table 1: Correlations between Flexible and Rigid Restraint and Preoccupying Cognitions

\begin{tabular}{lllcc}
\hline & \multicolumn{4}{c}{ Preoccupying cognitions } \\
& Shape & food & diet & Total \\
\hline $\begin{array}{c}\text { Flexible } \\
\text { restraint } \\
\text { Rigid restraint }\end{array}$ & 0.10 & -0.06 & $0.30^{* * *}$ & 0.11 \\
\hline
\end{tabular}

$* \mathrm{p}<0.05 ; * * \mathrm{p}<0.01 ; * * * \mathrm{p}<0.001$ 
Table 2: Results of $\mathrm{N}$-back working memory test

1-back

Number of Reaction time of correct responses

correct responses

(ms)

2-back

ID1 $(N=106)$

Mean \pm SD

$70.7 \pm 9.3$

$690 \pm 161$

with flexible

restraint

Correlation

with rigid

restraint

ID2 $(\mathrm{N}=73)$

Mean \pm SD

Correlation

with flexible

restraint

Correlation

with rigid

restraint

$*: p<0.05 ; \S: p<0.10$
$71.4 \pm 12.1$

0.07

0.03

0.04

$-0.03$

0.04

$66.0 \pm 5.4$

0.05

0.01

0.13
Number of correct responses

$0.18 \S$

Reaction time of correct responses

(ms)

$1042 \pm 265$

$0.19 \S$

0.00

0.14

$-0.12$

$962 \pm 234$

(

)

